JGG 2020;68:57-59

doi: 10.36150/2499-6564-342

\title{
The use of non-invasive ventilation to treat acute respiratory failure in long term care setting: clinical experience in elderly patient
}

\author{
Marta Clerici', Antonella Ferrari², Giorgio Gallimbeni², Luigi Cesare \\ Bergamaschini $^{3}$ \\ ${ }^{1}$ Geriatric School, University of Milan, Italy; ${ }^{2}$ Long term facility, A.S.P. Pio Albergo Trivulzio, Milan, \\ Italy; ${ }^{3}$ Department of Biomedical and Clinical Sciences "L. Sacco", University of Milan, A.S.P. Pio \\ Albergo Trivulzio, Milan, Italy
}

Background. Nowadays older patients with acute respiratory failure (ARF) leading to a severe respiratory acidosis are frequently ventilated using a non-invasive approach in intensive care unit (ICU). Current guidelines suggest that patients with more severe acidosis should be managed in a higher dependency area. However, the use of non-invasive ventilation (NIV) is effective and safe in treating ARF in general wards in elderly.

Case report. a 82 years old male patient was admitted to a long term care facility due to a severe chronic obstructive pulmonary disease (COPD). Three days after the admission, he suffered of severe acute exacerbation of COPD leading to hypercapnic coma. NIV in Bi-PAP mode and standard medical therapy was started, taking into consideration the do-not-intubate order of the patient. His state of consciousness improved after 48 hours of NIV with decreasing of $\mathrm{PaCO} 2$ values and the patient was progressively weaned from the ventilator.

Conclusions. NIV should be considered a successful approach even in unusual setting of long term facility. The choice of use NIV should be taken by multidisciplinary medical staff in a setting where nurses and medical team are trained in NIV protocol, in order to reduce ICU admission, intubation rates and mortality.

Key words: elderly, non-invasive ventilation, hypercapnic coma, long term care facility

How to cite this article: Clerici $M$, Ferrari A, Gallimbeni $G$, et al. The use of non-invasive ventilation to treat acute respiratory failure in long term care setting: clinical experience in elderly patient. Journal of Gerontology and Geriatrics 2020;68:57-9. https://doi.org/10.36150/2499-6564-342

(C) Copyright by Società Italiana di Gerontologia e Geriatria (SIGG)

\section{(c) (i) $(9)$}

\section{OPEN ACCESS}

This is an open access article distributed in accordance with the CC-BY-NC-ND (Creative Commons Attribution-NonCommercial-NoDerivatives 4.0 International) license. The article can be used by giving appropriate credit and mentioning the license, but only for non-commercial purposes and only in the original version. For further information: https://creativecommons.org/licenses/by-nc-nd/4.0/deed.en

\section{CASE DESCRIPTION AND DISCUSSION}

Here we report the case of a 82-years old male in hypercapnic coma treated successfully with non-invasive ventilation (NIV) in a long-term care facility. The patient was admitted because of a severe chronic obstructive pulmonary disease (COPD). In the two previous years, due to acute exacerbation of COPD he suffered of several severe episodes of acute respiratory failure (ARF) that required NIV and in one case tracheal intubation in Intensive Care Unit (ICU). His medical history included mild cognitive impairment without behavioral symptoms (Mini mental status examination: 25/30), ischemic heart disease, arterial hypertension, and benign hypertrophy prostate. 
Table I. Evolution of arterial blood gas values at the admission and during NIV.

\begin{tabular}{|c|c|c|c|c|c|c|}
\hline & $\begin{array}{l}\mathrm{FiO}_{2} \\
(\%)\end{array}$ & $\mathrm{pH}$ & $\begin{array}{c}\mathrm{pO}_{2} \\
(\mathrm{mmHg})\end{array}$ & $\underset{(\mathrm{mmHg})}{\mathrm{pCO}_{2}}$ & $\begin{array}{c}\mathrm{SpO}_{2} \\
(\%)\end{array}$ & $\begin{array}{c}\mathrm{HCO}_{3} \\
(\mathrm{mmol} / \mathrm{l})\end{array}$ \\
\hline Admission & 28 & 7.41 & 84 & 66 & 98.7 & 36.2 \\
\hline Start NIV & 28 & 7.33 & 64 & 92 & 90 & 39.2 \\
\hline 1 hour & 28 & 7.36 & 49 & 86 & 90 & 39.6 \\
\hline 6 hours & 28 & 7.36 & 64 & 97 & 94 & 43.7 \\
\hline 1 day & 36 & 7.41 & 49 & 77 & 89 & 40.3 \\
\hline 3 days & 32 & 7.23 & 66 & 131 & 96 & 41.3 \\
\hline 5 days & 32 & 7.38 & 72 & 95 & 97 & 45.5 \\
\hline 10 days & 30 & 7.5 & 92 & 48 & 100 & 39.6 \\
\hline 72 hours without NIV & 26 & 7.43 & 53 & 57 & 94 & 33.8 \\
\hline
\end{tabular}

At the admission in the long-term unit, the patient was alert, responsive and partially oriented. His Glasgow Coma Score (GCS) was 15. His arterial blood gases (ABG) tests are reported in Table I. On the third day, there was a rapidly worsening of his respiratory status. $\mathrm{ABG}$ showed slight respiratory acidosis $(\mathrm{pH}=7.33)$ with severe hypercapnia $\left(\mathrm{pCO}_{2}=92 \mathrm{mmHg}\right)$. NIV via facial mask was started using Bi-PAP mode: IPAP was $20 \mathrm{mmH}_{2} \mathrm{O}$ and EPAP was $6 \mathrm{mmH}_{2} \mathrm{O}$. The time of inspiration was $1.5 \mathrm{sec}$, respiratory rate was set at 16 breath/min and $\mathrm{FiO}_{2}$ was adjusted to maintain a $\mathrm{SaO}_{2}>90 \%$. After one hour of ventilation, $\mathrm{ABG}$ revealed a slight improvement in $\mathrm{pH}$ and $\mathrm{pCO}_{2}$. IPAP level was raised at $22 \mathrm{mmH}_{2} \mathrm{O}$ and standard medical therapy was started, including steroids iv and empirical antibiotic therapy with ceftriaxone.

The patient showed a moderate rejection to NIV due principally to facial mask discomfort and it was not possible to maintain proper ventilation. He became progressively agitated and confused, but no specific treatment was taken into consideration because of the lack of experience in sedation during NIV. His state of consciousness progressively worsened, until coma. His GCS was 5, with a severe respiratory acidosis $(\mathrm{pH}=7.23)$ and hypercapnia $\left(\mathrm{PaCO}_{2}=131 \mathrm{mmHg}\right)$ at $\mathrm{ABG}$. At this moment, invasive treatment should be started, but his relatives had refused intubation, respecting the patient's will. In front of this do-not-intubate (DNI) order, NIV was continued although the comatose state and after 48 hours of NIV his state of consciousness improved with decreasing of $\mathrm{PaCO}_{2}$ values. Regular pressure relief and skin-protective strategies were adopted to reduce patient's discomfort and improve NIV's effectiveness. The blood gas parameter remained acceptable and stable over time and the patient was progressively weaned from the ventilator.

Our aim is to support the possibility to apply NIV with success in a non-intensive setting. Nowadays older patients with ARF leading to a severe respiratory acidosis are frequently ventilated using a non-invasive approach in ICU ${ }^{1,2}$. However, the use of NIV on general wards has been investigated and in literature it is reported that NIV is effective and safe in treating ARF in a non-ICU setting in elderly followed by a multidisciplinary staff with appropriate experience ${ }^{3-6}$. Although current guidelines suggest that patients with more severe acidosis should be managed in a higher dependency area ${ }^{1}$, this case demonstrates that NIV should be considered a successful approach even in long term facility. In this setting the familiarity with the non invasive technique could define its success and the medical staff and nurses should be trained in NIV protocol to achieve the goal. In this contest, it could be possible to reduce ICU admission, intubation rates and mortality.

Furthermore, in presence of poor toleration to ventilation and comatose state, patients should be considered for intubation in ICU setting ${ }^{1,2}$. However, the decision to stop or continue NIV is not based on an objective physiological variable, but only on the patient's will and a review underlined the importance of NIV as the primary treatment when intubation is not wanted by the patient ${ }^{\text {. }}$. In this case of hypercapnic coma, the choice to continue NIV was supported by previous reports demonstrating that cautious application of NIV can be attempted in comatose patient by an experienced team ${ }^{8,9}$.

This case report showed that old patient affected by ARF and with DNI order could be treated successfully with NIV in an unusual setting of long term care facility, avoiding ICU admissions. The choice of NIV should be considered by a multidisciplinary medical staff in a setting where nurses and medical team are trained in NIV protocol.

\section{References}

1 British Thoracic Society Standards of Care Committee. 
Non-invasive ventilation in acute respiratory failure, British Thoracic Society Standards of Care Committee. Thorax 2002;57:192-211.

2 Conti G, Antonelli M, Navalesi P, et al. Noninvasive vs. conventional mechanical ventilation in patients with chronic obstructive pulmonary disease after failure of medical treatment in the ward: a randomized trial. Intensive Care Med 2002;28:1701-7.

3 Laudisio A, Scarlata S, Pedone C, et al. Noninvasive ventilation in older adults admitted to a pneumogeriatric unit. J Am Geriatr Soc 2014;62:1195-7.

4 La Regina M, Marinaro A, Scuotri L, et al. Non-invasive mechanical ventilation in internal medicine departments: a pilot study. Italian Journal of Medicine 2013;7:172-8.

5 Elliott MW, Confalonieri M, Nava S. Where to perform noninvasive ventilation? Eur Respir J 2002 19:1159-66.
6 Cabrini L, Antonelli M, Savoia G, et al. Non-invasive ventilation outside of the Intensive Care Unit: an Italian survey. Minerva Anestesiol 2011;77:313-22.

7 Piroddi I, Barlascini C, Esquinas A, et al. Non-invasive mechanical ventilation in elderly patients: a narrative review. Nicolini Geriatr Gerontol Int 2017;17:689-96.

8 Scala R, Nava S, Conti G, et al. Noninvasive versus conventional ventilation to treat hypercapnic encephalopathy in chronic obstructive pulmonary disease. Intensive Care Med 2007;33:2101-28. https://doi.org/10.1007/s00134007-0837-2

9 Gonzalez Dıaz G, Carrillo Alcaraz A, Pardo Talavera $\mathrm{JC}$, et al. Noninvasive positive-pressure ventilation to treat hypercapnic coma secondary to respiratory failure. Chest 2005;127:952-60. https://doi.org/10.1378/ chest.127.3.952 